\title{
Unidirectional HLA Mismatch
}

National Cancer Institute

\section{Source}

National Cancer Institute. Unidirectional HLA Mismatch. NCI Thesaurus. Code C126299.

The state when a recipient of a hematopoietic stem cell transplantation is not fully matched with their unrelated adult volunteer donor for HLA-A, HLA-B, HLA-C, and HLADRB1 and either the recipient or donor is heterozygous at one locus. Occurs when only the donor or recipient is homozygous. 